\title{
NO EVIDENCE FOR FREQUENCY-DEPENDENT SELECTION ACTING BETWEEN CLONES OF THE WATER FLEA, DAPHNIA MAGNA
}

\author{
J. F. Y. BROOKFIELD* \\ Genetics Department, University College of Swansea, Singleton Park, \\ Swansea SA2 8PP, U.K.
}

Received 18.ix.80

\section{SUMMARY}

Competition experiments were carried out between clones of the water flea, Daphnia magna, in an effort to detect frequency-dependent selection maintaining clonal coexistence. Pairs of clones were used, both from the same and from different sources. No stable equilibrium was detected, but in the first series of experiments, in which gene frequencies were assessed by the electrophoresis of samples, it was found that differences in the level of sexuality between clones were resulting in artificial selection.

A second series of experiments involved competition between morphologically distinguishable clones. An analysis of the computed selection acting on the populations, and reproductive rate differences, failed to show significant effects of clone frequency on these quantities in the experiments. There was some evidence that reproductive rate differences between clones were selectively important.

\section{INTRODUCTION}

ONE type of selection that may be important in maintaining the observed genetic diversity of populations is frequency-dependent selection, whereby genotypes increase their fitness as they become rare (Ayala and Campbell, 1974). The best experimental evidence for such selection in wild populations comes from situations where phenotypic differences accompany the genetic polymorphism, as in mimicry (Brower et al., 1967), and "apostatic" selection, whereby predators, of which the most studied have been ground feeding passerines, preferentially eat common morphs when confronted by a polymorphic prey species (Clarke, 1962; Allen and Clarke, 1968; Allen, 1972).

However, most known polymorphisms are defined biochemically, and for these the mechanism of frequency-dependent selection must be different, although it is possible that molecular mimicry between hosts and parasites may play a part in the maintenance of some polymorphisms (Clarke, 1975, 1976). Theoretical studies indicate that genetic polymorphism stabilised by frequency-dependent selection can arise through niche diversification, with selection acting in different directions in different niches (Levene, 1953), but only if the selection is soft (Dempster, 1955; Wallace, 1968). Considerable theoretical development of these ideas has taken place, but in panmictic conditions the robustness of the polymorphic equilibria generated is low (Hedrick et al., 1976; Hoekstra, 1978; Maynard Smith and Hoekstra, 1980). Models of this kind are usually worded in terms of spatial environmental heterogeneity, but their prediction of "protected" polymorphism (that is, polymorphism where fixation of any allele is unstable

* Present address: Laboratory of Genetics, N.I.E.H.S., P.O. Box 12233, Research Triangle Park, North Carolina 27709, U.S.A. 
(Prout, 1968)) with panmixia, means that they are mathematically equivalent to models in which the environment heterogeneity envisaged is not spatial. The heterogeneity could, for example, consist of a range of available food types, which different genotypes utilise with differing efficiencies.

The experimental demonstration of frequency-dependent selection is faced with the problem that the change in gene frequencies in panmictic sexual populations under the influence of heterozygote advantage is very similar to that predicted in a frequency-dependent selection model. Statistical treatments of observed changes in gene frequency showing that a model including frequency-dependent fitness values gives a better fit to data than a heterozygote advantage model (Wright and Dobzhansky, 1946; Spiess, 1957; Tobari and Kojima, 1967), do not resolve the issue categorically as some improvement in fit inevitably arises from the increased number of degrees of freedom in the "frequency-dependent" models. Furthermore, frequency-dependent mating success (Spiess, 1970; Ehrman, 1966; Ehrman et al., 1965) could maintain a polymorphism even in the absence of frequency-dependent viability effects.

One way in which frequency-dependent selection can be unambiguously distinguished from heterozygote advantage is by looking at the fitnesses of genotypes over only part of the life cycle, to see if such fitnesses vary with genotypic frequencies. Kojima and Yarborough (1967), Morgan (1976) and Birley and Beardmore (1977) looked for frequency-dependent variation in egg-to-adult survivorship in crowded cultures of Drosophila melanogaster and found evidence for strong frequency-dependence. The survivorships of the genotypes at the esterase- 6 locus were higher when they were rare than when common. The same effect was found in studies of Drosophila melanogaster genotypes differing at the alcohol dehydrogenase (ADH) locus (Kojima and Tobari, 1969a; Morgan, 1976) and of Drosophila ananassae differing by the chromosome 2 inversions L-A and L-B (Kojima and Tobari, 1969b). Later experiments showed frequency-dependence in egg-to-adult viabilities for genotypes differing at the Payne inversion in D. melanogaster (Nassar et al., 1973) and very strong frequency-dependence in the same measure at the leucine amino peptidase (LAP) locus (Nassar, 1979).

Egg-to-adult viability measurements require strong selection in favour of a rare morph before such selection can be significantly demonstrated in samples of reasonable size, but if the egg-to-adult survivals of samples vary significantly with frequency then the frequency-dependence of selection could be established even if selection is not significantly demonstrated in any individual sample. Snyder and Ayala (1979) found evidence of frequency-dependence in both rate of development and egg-to-adult survival at the phosphoglucomutase (PGM)-1 locus in D. pseudoobscura and Marinkovic and Ayala (1975) found these effects at the malic enzyme locus. However, Yoshimaru and Mukai (1979) detected no frequency-dependence at the ADH locus in D. melanogaster and Yamazaki (1971) found no such selection acting on the esterase-5 locus in $D$. pseudoobscura.

One means by which the frequency-dependence observed in such experiments may be produced is by larvae conditioning the food medium in a way more harmful to others of their genotype than to larvae of other genotypes. Experiments by Huang, Singh and Kojima (1971) showed that 
if larvae of one genotype at the esterase- 6 locus in $D$. melanogaster are used to condition media in which other larvae are then grown and tested for survival, the rate of survival of larvae of the same genotype as the conditioning genotype is less than that of the other larvae. Kojima and Huang (1972) found this effect to be dependent upon density, being manifest only when the experimental larvae were crowded. Dolan and Robertson (1975), however, found no evidence for this effect at either the $\mathrm{ADH}$ or the esterase locus under crowded conditions.

In these experiments stabilising selection occurs in the absence of diversity in the food supply. Clarke (1979) and Clarke and Allendorf (1979) have produced a model whereby genotypes producing allozymes differing in their kinetic constants might be expected to show frequencydependent selection in conditions where the enzymes' substrates are limiting for population growth. Evidence for biochemical differences between allozymes is strong for the $D$. melanogaster esterase- 6 locus (Danford and Beardmore, 1979), the ADH locus (Day et al., 1974; Ward, 1974; Gibson, 1970 ), the $D$. virilis $\alpha$ - and $\beta$-esterases (Narise, 1971, 1973), the esterase- 1 locus in Catastomus clarkii (Koehn, 1969) and 16 out of 23 polymorphic human loci studied (Harris, 1971). The implication of these results is that frequency-dependent selection might be expected to act between almost any pair of alleles. Stewart and Levin (1973), in a model mathematically similar to Clarke and Allendorf's, predict that genotypes with different resource uptake functions can coexist on a single resource, if that resource is supplied occasionally in large quantities, not constantly. However, the robustness of these models is very low (Maynard Smith and Hoekstra, 1980).

If these theories are correct, however, randomly chosen genotypes should show some frequency-dependent selection with respect to each other. Genotypes from the same wild populations, moreover, are more likely to show frequency-dependent selection. Their coexistence should be favoured if they show frequency-dependent selection in the wild.

Daphnia magna Straus, a member of the crustacean suborder Cladocera, reproduces mainly by apomictic parthenogenesis. In this process, a variable number of eggs are laid into the brood pouch which develop into swimming embryos, which are usually female and which are released after 2-3 days (Green, 1954; Obreshkove and Fraser, 1940; Anderson and Jenkins, 1942). Sexual reproduction also occurs in which females produce two eggs which are released at the next moult, to develop into female offspring after a period of diapause which may last weeks, months, or in some cases, years.

The parthenogenetic eggs of Cladocera are produced without meiosis, as shown by the absence of segregation of electrophoretic markers in Daphnia magna (Hebert and Ward, 1972), D. pulex (Berger and Sutherland, 1978) and Bosmina longirostris (Manning et al., 1978). It is probable that the offspring and parents were genetically identical, although Banta, Wood, Brown and Ingle (1939) were able successfully to select for the characters of "sex intergrade" and "excavated head" in clones of D. longispina and "sex intergrade" in the related Simocephalus exspinosus. In the parthenogenetic phase of aphids, selection for esterase activity (Bunting and Van Emden, 1980) and insecticide resistance (Berenek, 1974) can be successful, although again electrophoretically-defined heterozygotes 
do not segregate (Suomalainen et al., 1980). The supposed genetic identity or near identity of the Cladoceran parthenogens means that large clones of known genotype can be established and used in the study of frequencydependent selection.

Cladoceran populations contain considerable genetic diversity as measured by gel electrophoresis. This is true of D. magna (Hebert, 1972, $1974 a, 1974 b, 1974 c, 1975,1978$; Hebert and Ward, 1976; Hebert et al., 1972; Young, 1975, 1979a, 1979b), D. pulex (Berger and Sutherland, 1978; Hebert and Crease, 1980), Bosmina longirostris (Manning et al., 1978) and Simocephalus serrulatus (Smith and Fraser, 1976). Heterozygote advantage would not, in a population where sexuality is rare, maintain great genetic diversity, but rather should cause the near-fixation of a multiply heterozygous clone (Berger, 1976; but see Angus, 1978). In Canadian populations of $D$. pulex reproducing purely asexually, as many as seven genotypes (as defined by 11 electrophoretic loci) have been found to coexist, and a study of larger numbers using more loci would almost certainly reveal an even larger number (Hebert and Crease, 1980). The diversity of, and the lack of consistent heterozygote excesses in Cladoceran populations constitute weak evidence that genetic variability is maintained by frequencydependent selection.

Cladocera can be used in the laboratory to test for such selection, which is the only process capable of causing the stable coexistence of clones. If frequency-dependent selection is a general phenomenon, the frequencydependent effects of the many loci at which two clones differ may summate and thus weak-per-locus selection may be reflected as strong interclonal selection. Experiments were carried out using clones both from different sources and from the same pond.

\section{MATERIALS AND METHODS}

The following clones were used:

Clone A. From Regents Park Pond (0.5. Map 176. Grid Reference 283825). 2.12.1976. Genotype: Est-1 :SS GOT:FF MDH:MM.

Clone B. From Regents Park Pond $(0 \cdot 5$. Map 176. Grid Reference 283825). 21.4.1977. Genotype: Est-1 :SS GOT:FS MDH:MM.

Clone C. Bought from Childes Pet Shop, Caledonian Road. 20.5.1977. Genotype: Est-1:SK GOT:FF MDH:MM.

Clone D. From Regents Park Pond $(0 \cdot 5$. Map 176. Grid Reference 283825). 24.6.1977. Genotype: Est-1 : SS GOT:SS MDH:MF.

Clone E. From Regents Park Pond $(0 \cdot 5$. Map 176. Grid Reference 283825). 25.6.1977. Genotype: Est-1 :MM GOT:SF MDH:MM.

Clone F. Offspring of a sexual cross between two from clone E. 1.12.1977. Genotype: Est-1 :MM GOT:SF MDH: Unknown (Presumably MM).

Clone G. White-eyed clone from Imperial College. Received 27.8.1977. Genotype: Est-1:SF GOT:FF MDH:MM.

Electrophoresis was carried out with gels of volume $350 \mathrm{ml}$, of 12 per cent starch, using the gel and electrode buffers of Kristjansson (1963), and a voltage of 350 volts for 2 hours at $10^{\circ} \mathrm{C}$. The gels were sliced and stained for the following enzyme activities:

Esterase: The stain was $20 \mathrm{mg}$. Alpha-naphthylacetate, $150 \mathrm{mg}$ Fast Red TR salt, $150 \mathrm{ml}$ Phosphate Buffer at $p \mathrm{H} 6.5$ (which was $20.8 \mathrm{~g}$ $\mathrm{NaH}_{2} \mathrm{PO}_{4} \cdot 2 \mathrm{H}_{2} \mathrm{O}+9 \cdot 47 \mathrm{~g} \mathrm{Na}_{2} \mathrm{HPO}_{4}$ in 1 litre $\mathrm{H}_{2} \mathrm{O}$ ). The $20 \mathrm{mg}$ of ester was 
dissolved in $5 \mathrm{ml}$ of a 1:1 mixture of acetone and water. Only one locus (Est-1) produced strong, repeatable bands on the gels. The alleles were named following the terminology of Young (1975).

Glutamate Oxaloacetate Transaminase (GOT): The stain was $0.6 \mathrm{mg}$. Pyridoxal 5' Phosphate, $0.276 \mathrm{~g} \mathrm{~L}$-aspartic acid, 0.156 g Alpha-ketoglutaric acid and $0.24 \mathrm{~g}$ Fast Blue $\mathrm{BB}$ salt in $60 \mathrm{ml}$ Phosphate Buffer at $p \mathrm{H} 7.5$ (which was $28.4 \mathrm{~g} \mathrm{Na}_{2} \mathrm{HPO}_{4}$ in 1 litre $\mathrm{H}_{2} \mathrm{O}$ adjusted to $p \mathrm{H} 7.5$ in $1 \mathrm{~N} \mathrm{HCl}$ and readjusted to $p \mathrm{H} 7.5$ with $1 \mathrm{~N} \mathrm{NaOH}$ following addition of all compounds except the Fast Blue BB salt). The alleles were named following the terminology of Young (1975).

Malate Dehydrogenase (MDH): The stain was $1.56 \mathrm{~g} \mathrm{Malic} \mathrm{acid,} 80 \mathrm{mg}$ Nicotinamide adenine dinucleotide, $20 \mathrm{mg}$ Thiazolyl Blue and $20 \mathrm{mg}$ Phenazine Methosulphate in $100 \mathrm{ml}$ Tris- $\mathrm{HCl}$ Buffer at $p \mathrm{H} 8.0$ (which was $24.23 \mathrm{~g}$ Tris (hydroxymethyl) methylamine adjusted to $p \mathrm{H} 8.0$ with $1 \mathrm{~N}$ $\mathrm{HCl}$ and readjusted to $p \mathrm{H} 8.0$ with $1 \mathrm{~N} \mathrm{NaOH}$ following addition of the Malic acid). The alleles were named following the terminology of Hebert and Ward (1972).

Artificial environments were established in the laboratory in glass jars by the addition of 50 grams of soil to $100 \mathrm{~cm}^{3}$ of pond water (from the pond from which some of the Daphnia used in the experiment were obtained) and distilled water to a volume of $2 \frac{1}{2}$ litres (except for experiments 5 and 6 in which $1 \frac{1}{2}$ litres were used). After one day a total of 50 newborn females from two clones were added. The jars were placed indoors in daylight. For experiments 1-10, each week the numbers in each of three categories were counted. These were:

(1) Young;

(2) Adults without ephippia;

(3) Adult females with ephippia.

Each day any females with ephippia were removed and examined electrophoretically at the Est-1 locus. For experiments 11-18 all ephippial females were removed but not electrophoresed, the populations were counted and the sex of any adults and the number of parthenogenetic eggs they possessed were recorded. Counting of the cultures was less regular in these experiments.

In experiments 1-11 some non-sexual females were removed, and in 1-10 electrophoresed. Prior to the population size reaching 300 individuals, ten adult females were removed, and subsequently 20 adult females were removed at the end of each week if less than 20 ephippial females had been removed during the week.

The clones were founded from single females and kept under similar conditions to those used in the experiments and with a protocol of the removal of females similar to that followed in the experimental populations. In heterozygous clones no segregation of homozygotes was observed in these stock cultures. Thus the clones in the experimental cultures should have remained pure. The young females used initially are shown in table 1 .

\section{Results}

From the results of the electrophoresis carried out in experiments 1-10, 2 -week running averages were obtained for the proportions of each clone. 
TABLE 1

Initial compositions of the experiments

$\begin{array}{ll}\text { Experiment } & 1 \\ \text { Experiment } & 2 \\ \text { Experiment } & 3 \\ \text { Experiment } & 4 \\ \text { Experiment } & 5 \\ \text { Experiment } & 6 \\ \text { Experiment } & 7 \\ \text { Experiment } & 8 \\ \text { Experiment } & 9 \\ \text { Experiment } 10 \\ \text { Experiment } 11 \\ \text { Experiment } 12 \\ \text { Experiment } 13 \\ \text { Experiment } 14 \\ \text { Experiment } 15 \\ \text { Experiment } 16 \\ \text { Experiment } 17 \\ \text { Experiment } 18\end{array}$

45 Clone C: 5 Clone B 45 Clone $\mathrm{B}$ : 5 Clone $\mathrm{C}$ 45 Clone C: 5 Clone B 45 Clone B: 5 Clone $\mathrm{C}$ 45 Clone C: 5 Clone A 45 Clone A: 5 Clone $\mathrm{C}$ 45 Clone C: 5 Clone A 45 Clone A: 5 Clone $\mathrm{C}$ 45 Clone E: 5 Clone D 45 Clone D: 5 Clone E 25 Clone C: 25 Clone $\mathrm{G}$ 25 Clone C: 25 Clone G 25 Clone C: 25 Clone $\mathrm{G}$ 25 Clone C: 25 Clone G 25 Clone A: 25 Clone $G$ 25 Clone A: 25 Clone $G$ 25 Clone $\mathrm{G}$ : 25 Clone $\mathrm{G}$ 25 Clone A: 25 Clone $\mathrm{G}$

The variations in such sample proportions with time are shown in fig. 1-3. The competition between clones $\mathrm{B}$ and $\mathrm{C}$ (which came from different sources) in experiments 1-4 always resulted in the fixation of clone $C$. Competition between $\mathrm{A}$ and $\mathrm{C}$ (which again came from different sources) resulted in the fixation of clone $\mathrm{C}$ in experiments 5 and 7 (when it initially formed 90 per cent of the population) and a large increase in its frequency in experiments 6 and 8 (when it initially formed 10 per cent of the population). In experiments 9 and 10 clone $\mathrm{D}$ very rapidly replaced clone E from the same source. Thus there does not appear to be any intermediate stable equilibrium frequency with these clones. These frequency estimates

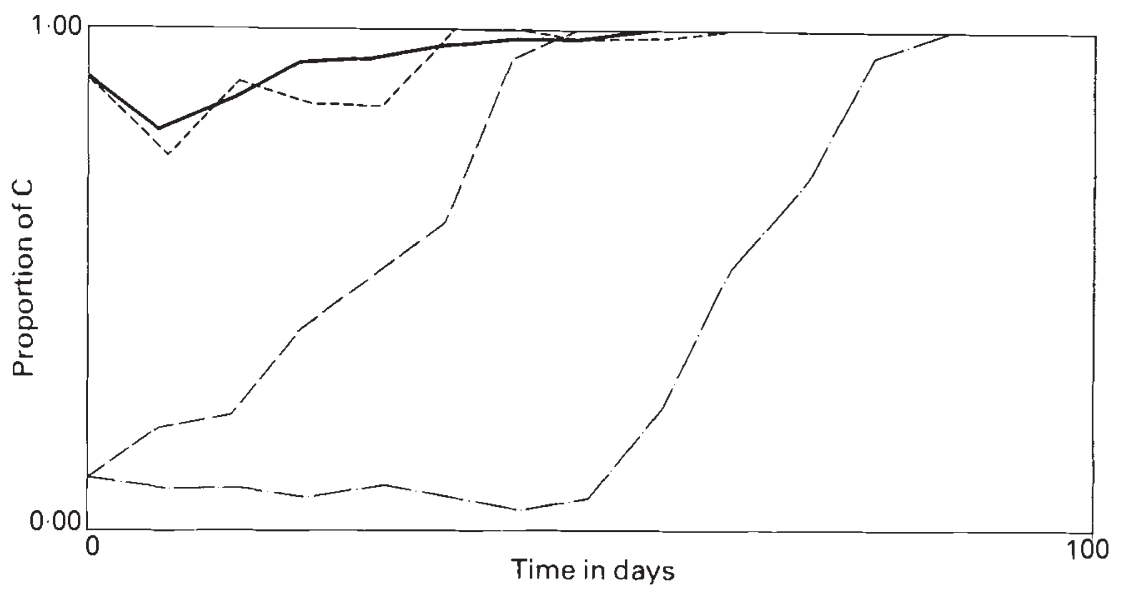

FIG. 1.-Variations in the proportions of clone $\mathrm{C}$ in competition with clone $\mathrm{B}$ in experiments 1-4 against time in days. The values are two-week running averages. Clone $\mathrm{C}$ initially comprised 90 per cent of the population in experiments 1 and 3 and 10 per cent in experiments 2 and 4 . The average sample size used in these values is 55, giving a standard error of 0.067 in $q$ when the clones have equal frequencies. $-=$ Exper 


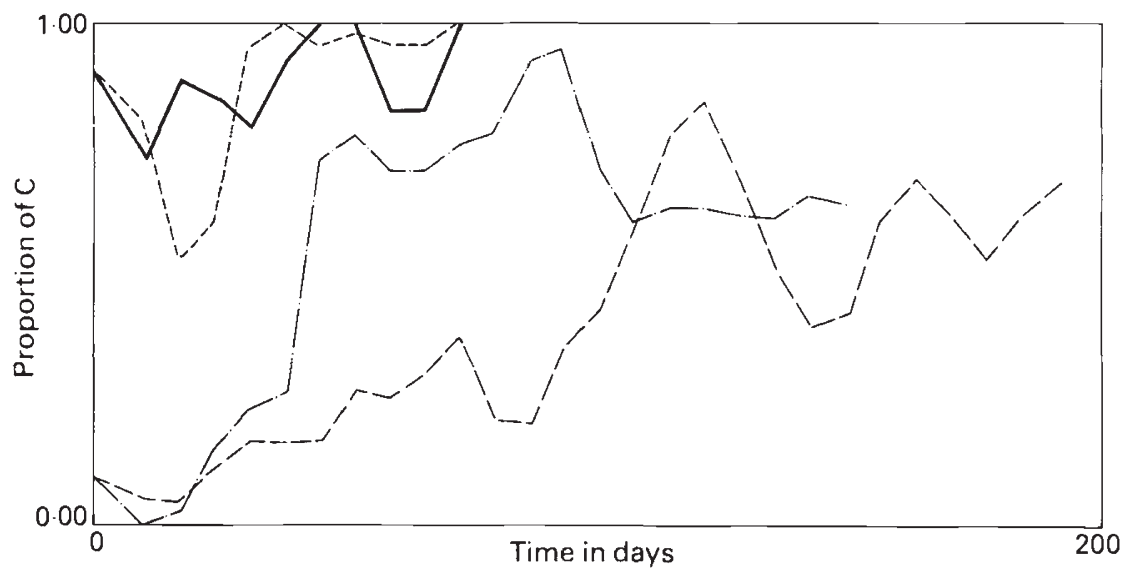

FIG. 2.-Variation in the proportions of clone $A$ in competition with clone $B$ in experiments 5-8 against time in days. Clone $C$ initially comprised 90 per cent of the population in experiments 5 and 7 and 10 per cent in experiments 6 and 8 . The average sample size used in these values is 45 , giving a standard error of 0.074 in $q$ when the clones have equal frequencies. $-=$ Experiment $5,---=$ Experiment $6,----=$ Experiment $7, \cdot-\cdot-\cdot-=$ Experiment 8 .

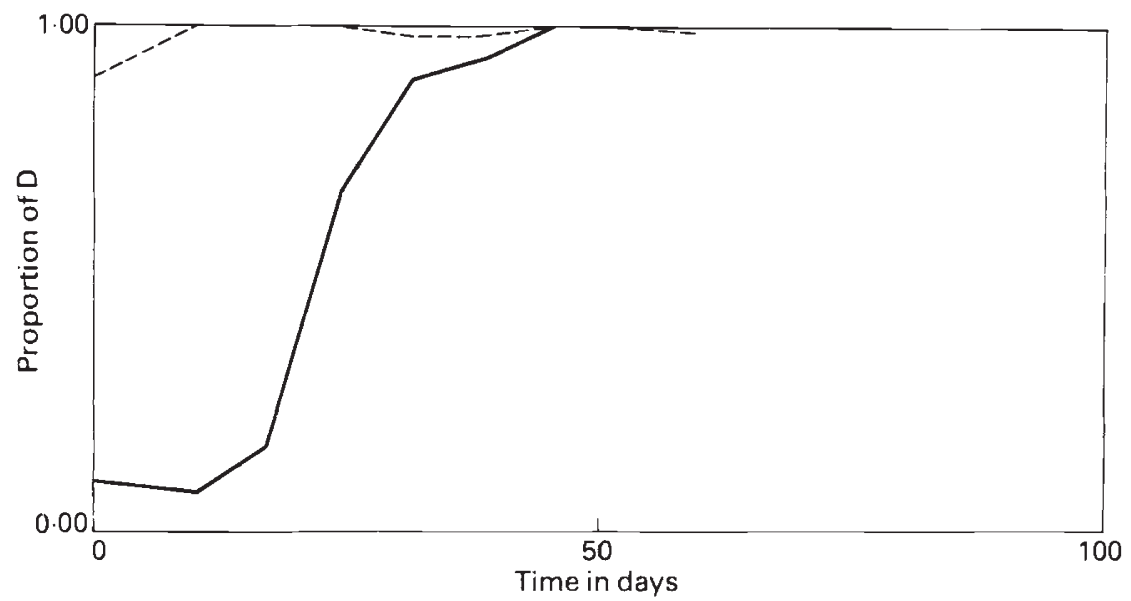

FIG. 3.-Variations in the proportions of clone $D$ in competition with clone $E$ in experiments 9 and 10 against time in days. Clone $D$ initialiy comprised 10 per cent of the population in experiment 9 and 90 per cent in experiment 10. The average sample size used in these values is 74 , giving a standard error of 0.058 in $q$ when the clones have equal frequencies. $-=$ Experiment $9,---=$ Experiment 10.

were based on destructive sampling without replacement of adults from the population, the majority of which were sexual. Sexual females had to be removed in order to prevent the addition to the population of ephippia and sexually derived offspring (which can, on occasion, hatch without diapause (Wood, 1932; Hebert, 1978)) with recombined genotypes and unpredictable selective characteristics. Sexual females removed were not replaced as it was felt that their replacement with females with a level of 
parthenogenetic egg production different from that characterising the experimental cultures would have a greater effect on clone frequencies than the protocol of non-replacement. However, removal of all ephippial females had the effect that if the clones differed in their degrees of sexuality not only would the clone with the higher level of sexuality be overrepresented in the sexual portions of the samples but also such a clone would suffer artificial selection against it. Tables 2 and 3 show that in experiments 1-4 and experiments 5-8 in every case when there was a significant difference in the proportions of clone $\mathrm{C}$ in sexual and non-sexual samples removed from the same population in the same week, clone $C$ was underrepresented in the sexual population. Thus the success of clone $\mathrm{C}$ in competition with clones $\mathrm{A}$ and $\mathrm{B}$ is partially due to artificial selection applied through the protocol of the removal of ephippial females.

\section{TABLE 2}

Comparison of numbers of different genotypes in sexual and asexual samples (Experiments 1 , $2,3,4)$

\begin{tabular}{|c|c|c|c|c|c|c|c|c|c|}
\hline \multirow[t]{2}{*}{ Week } & \multirow[t]{2}{*}{ Type } & \multicolumn{4}{|c|}{$\begin{array}{l}\text { Genotype C } \\
\text { Experiments }\end{array}$} & \multicolumn{4}{|c|}{$\begin{array}{l}\text { Genotype B } \\
\text { Experiments }\end{array}$} \\
\hline & & 1 & 2 & 3 & 4 & 1 & 2 & 3 & 4 \\
\hline 1 & $\begin{array}{c}\text { Sexual } \\
\text { Asexual }\end{array}$ & $\begin{array}{l}0 \\
8\end{array}$ & $\begin{array}{l}0 \\
2\end{array}$ & $\begin{array}{r}2 \\
10\end{array}$ & $\begin{array}{l}0 \\
1\end{array}$ & $\begin{array}{l}0 \\
2\end{array}$ & $\begin{array}{l}0 \\
8\end{array}$ & $\begin{array}{l}4^{* * *} \\
0\end{array}$ & $\begin{array}{l}3 \\
9\end{array}$ \\
\hline 2 & $\begin{array}{l}\text { Sexual } \\
\text { Asexual }\end{array}$ & $\begin{array}{l}1 \\
9\end{array}$ & $\begin{array}{l}2 \\
5\end{array}$ & $\begin{array}{l}12 \\
10\end{array}$ & $\begin{array}{l}1 \\
2\end{array}$ & $\begin{array}{l}0 \\
1\end{array}$ & $\begin{array}{c}17^{* *} \\
5\end{array}$ & $\begin{array}{l}0 \\
0\end{array}$ & $\begin{array}{r}25 \\
8\end{array}$ \\
\hline 3 & $\begin{array}{c}\text { Sexual } \\
\text { Asexual }\end{array}$ & $\begin{array}{r}73 \\
0\end{array}$ & $\begin{array}{r}23 \\
0\end{array}$ & $\begin{array}{r}5 \\
19\end{array}$ & $\begin{array}{l}0 \\
0\end{array}$ & $\begin{array}{l}5 \\
0\end{array}$ & $\begin{array}{r}24 \\
0\end{array}$ & $\begin{array}{l}7^{* * * *} \\
1\end{array}$ & $\begin{array}{r}3 \\
10\end{array}$ \\
\hline 4 & $\begin{array}{c}\text { Sexual } \\
\text { Asexual }\end{array}$ & $\begin{array}{r}61 \\
0\end{array}$ & $\begin{array}{r}28 \\
0\end{array}$ & $\begin{array}{r}1 \\
20\end{array}$ & $\begin{array}{l}0 \\
2\end{array}$ & $\begin{array}{l}3 \\
0\end{array}$ & $\begin{array}{r}27 \\
0\end{array}$ & $\begin{array}{l}0 \\
0\end{array}$ & $\begin{array}{l}0 \\
8\end{array}$ \\
\hline 5 & $\begin{array}{c}\text { Sexual } \\
\text { Asexual }\end{array}$ & $\begin{array}{r}26 \\
0\end{array}$ & $\begin{array}{r}17 \\
0\end{array}$ & $\begin{array}{r}0 \\
20\end{array}$ & $\begin{array}{l}0 \\
0\end{array}$ & $\begin{array}{l}0 \\
0\end{array}$ & $\begin{array}{l}2 \\
0\end{array}$ & $\begin{array}{l}0 \\
0\end{array}$ & $\begin{array}{l}13 \\
10\end{array}$ \\
\hline 6 & $\begin{array}{c}\text { Sexual } \\
\text { Asexual }\end{array}$ & $\begin{array}{l}10 \\
16\end{array}$ & $\begin{array}{r}3 \\
13\end{array}$ & $\begin{array}{r}0 \\
20\end{array}$ & $\begin{array}{l}2 \\
4\end{array}$ & $\begin{array}{l}0 \\
1\end{array}$ & $\begin{array}{l}0 \\
0\end{array}$ & $\begin{array}{l}0 \\
0\end{array}$ & $\begin{array}{c}116^{* * * * *} \\
6\end{array}$ \\
\hline 7 & $\begin{array}{c}\text { Sexual } \\
\text { Asexual }\end{array}$ & $\begin{array}{r}0 \\
20\end{array}$ & $\begin{array}{r}0 \\
16\end{array}$ & $\begin{array}{l}20 \\
21\end{array}$ & $\begin{array}{l}0 \\
4\end{array}$ & $\begin{array}{l}0 \\
0\end{array}$ & $\begin{array}{l}0 \\
0\end{array}$ & $\begin{array}{l}0 \\
1\end{array}$ & $\begin{array}{c}13^{* *} \\
6\end{array}$ \\
\hline 8 & $\begin{array}{c}\text { Sexual } \\
\text { Asexual }\end{array}$ & $\begin{array}{r}0 \\
42\end{array}$ & $\begin{array}{r}0 \\
41\end{array}$ & $\begin{array}{r}0 \\
20\end{array}$ & $\begin{array}{l}0 \\
4\end{array}$ & $\begin{array}{l}0 \\
0\end{array}$ & $\begin{array}{l}0 \\
1\end{array}$ & $\begin{array}{l}0 \\
0\end{array}$ & $\begin{array}{l}1 \\
6\end{array}$ \\
\hline 9 & $\begin{array}{l}\text { Sexual } \\
\text { Asexual }\end{array}$ & & & $\begin{array}{r}3 \\
20\end{array}$ & $\begin{array}{r}0 \\
20\end{array}$ & & & $\begin{array}{l}0 \\
0\end{array}$ & $\begin{array}{l}15^{* * * *} \\
0\end{array}$ \\
\hline 10 & $\begin{array}{c}\text { Sexual } \\
\text { Asexual }\end{array}$ & & & $\begin{array}{r}16 \\
0\end{array}$ & $\begin{array}{r}1 \\
17\end{array}$ & & & $\begin{array}{l}0 \\
0\end{array}$ & $\begin{array}{l}1^{*} \\
1\end{array}$ \\
\hline 11 & $\begin{array}{l}\text { Sexual } \\
\text { Asexual }\end{array}$ & & & $\begin{array}{r}15 \\
1\end{array}$ & $\begin{array}{r}0 \\
16\end{array}$ & & & $\begin{array}{l}0 \\
0\end{array}$ & $\begin{array}{l}0 \\
0\end{array}$ \\
\hline 12 & $\begin{array}{l}\text { Sexual } \\
\text { Asexual }\end{array}$ & & & $\begin{array}{r}0 \\
12\end{array}$ & $\begin{array}{r}0 \\
20\end{array}$ & & & $\begin{array}{l}0 \\
0\end{array}$ & $\begin{array}{l}0 \\
0\end{array}$ \\
\hline 13 & $\begin{array}{l}\text { Sexual } \\
\text { Asexual }\end{array}$ & & & & $\begin{array}{r}0 \\
18\end{array}$ & & & & $\begin{array}{l}0 \\
0\end{array}$ \\
\hline 14 & $\begin{array}{l}\text { Sexual } \\
\text { Asexual }\end{array}$ & & & & $\begin{array}{r}0 \\
20\end{array}$ & & & & $\begin{array}{l}0 \\
0\end{array}$ \\
\hline
\end{tabular}

Any figure marked with one or more stars indicates that the number of sexual individuals is higher than would be expected by chance as measured by a chi-squared test at ${ }^{*}: P=0 \cdot 05$, **: $P=0.02, * * *: P=0.01,{ }^{* * * *}: P=0.001$. 
TABLE 3

Comparison of numbers of different genotypes in sexual and asexual samples (Experiments 5 , $6,7,8$ )

\begin{tabular}{|c|c|c|c|c|c|c|c|c|c|}
\hline \multirow[t]{2}{*}{ Week } & \multirow[t]{2}{*}{ Type } & \multicolumn{4}{|c|}{$\begin{array}{l}\text { Genotype C } \\
\text { Experiments }\end{array}$} & \multicolumn{4}{|c|}{$\begin{array}{l}\text { Genotype A } \\
\text { Experiments }\end{array}$} \\
\hline & & 5 & 6 & 7 & 8 & 5 & 6 & 7 & 8 \\
\hline 1 & $\begin{array}{c}\text { Sexual } \\
\text { Asexual }\end{array}$ & $\begin{array}{l}0 \\
8\end{array}$ & $\begin{array}{l}0 \\
1\end{array}$ & $\begin{array}{l}0 \\
8\end{array}$ & $\begin{array}{l}0 \\
0\end{array}$ & $\begin{array}{l}1 \\
2\end{array}$ & $\begin{array}{r}10 \\
7\end{array}$ & $\begin{array}{l}0 \\
2\end{array}$ & $\begin{array}{r}0 \\
10\end{array}$ \\
\hline 2 & $\begin{array}{c}\text { Sexual } \\
\text { Asexual }\end{array}$ & $\begin{array}{r}40 \\
6\end{array}$ & $\begin{array}{l}0 \\
1\end{array}$ & $\begin{array}{r}10 \\
9\end{array}$ & $\begin{array}{l}0 \\
1\end{array}$ & $\begin{array}{l}4 \\
0\end{array}$ & $\begin{array}{r}22 \\
8\end{array}$ & $21^{* * *}$ & $\begin{array}{r}21 \\
9\end{array}$ \\
\hline 3 & $\begin{array}{c}\text { Sexual } \\
\text { Asexual }\end{array}$ & $\begin{array}{r}28 \\
5\end{array}$ & $\begin{array}{r}11 \\
4\end{array}$ & $\begin{array}{r}19 \\
0\end{array}$ & $\begin{array}{l}9 \\
2\end{array}$ & $\begin{array}{l}8 \\
2\end{array}$ & $\begin{array}{l}89^{* * * *} \\
5\end{array}$ & $\begin{array}{l}3 \\
0\end{array}$ & $\begin{array}{r}30 \\
8\end{array}$ \\
\hline 4 & $\begin{array}{c}\text { Sexual } \\
\text { Asexual }\end{array}$ & $\begin{array}{r}0 \\
17\end{array}$ & $\begin{array}{r}14 \\
0\end{array}$ & $\begin{array}{r}36 \\
0\end{array}$ & $\begin{array}{l}5 \\
0\end{array}$ & $\begin{array}{l}0 \\
3\end{array}$ & $\begin{array}{r}52 \\
0\end{array}$ & $\begin{array}{l}0 \\
0\end{array}$ & $\begin{array}{r}16 \\
0\end{array}$ \\
\hline 5 & $\begin{array}{c}\text { Sexual } \\
\text { Asexual }\end{array}$ & $\begin{array}{l}0 \\
0\end{array}$ & $\begin{array}{l}2 \\
0\end{array}$ & $\begin{array}{l}0 \\
0\end{array}$ & $\begin{array}{l}0 \\
0\end{array}$ & $\begin{array}{l}0 \\
0\end{array}$ & $\begin{array}{r}31 \\
0\end{array}$ & $\begin{array}{l}0 \\
0\end{array}$ & $\begin{array}{l}0 \\
0\end{array}$ \\
\hline 6 & $\begin{array}{c}\text { Sexual } \\
\text { Asexual }\end{array}$ & $\begin{array}{r}0 \\
20\end{array}$ & $\begin{array}{l}1 \\
6\end{array}$ & $\begin{array}{r}0 \\
19\end{array}$ & $\begin{array}{r}0 \\
15\end{array}$ & $\begin{array}{l}0 \\
0\end{array}$ & $\begin{array}{r}1 \\
14\end{array}$ & $\begin{array}{l}0 \\
1\end{array}$ & $\begin{array}{l}0 \\
5\end{array}$ \\
\hline 7 & $\begin{array}{c}\text { Sexual } \\
\text { Asexual }\end{array}$ & $\begin{array}{r}0 \\
20\end{array}$ & $\begin{array}{l}0 \\
4\end{array}$ & $\begin{array}{r}0 \\
20\end{array}$ & $\begin{array}{r}0 \\
16\end{array}$ & $\begin{array}{l}0 \\
0\end{array}$ & $\begin{array}{r}0 \\
16\end{array}$ & $\begin{array}{l}0 \\
0\end{array}$ & $\begin{array}{l}0 \\
4\end{array}$ \\
\hline 8 & $\begin{array}{c}\text { Sexual } \\
\text { Asexual }\end{array}$ & $\begin{array}{r}0 \\
13\end{array}$ & $\begin{array}{l}0 \\
6\end{array}$ & $\begin{array}{l}0 \\
0\end{array}$ & $\begin{array}{l}0 \\
8\end{array}$ & $\begin{array}{l}0 \\
7\end{array}$ & $\begin{array}{r}0 \\
14\end{array}$ & $\begin{array}{l}0 \\
1\end{array}$ & $\begin{array}{l}0 \\
6\end{array}$ \\
\hline 9 & $\begin{array}{c}\text { Sexual } \\
\text { Asexual }\end{array}$ & $\begin{array}{r}0 \\
20\end{array}$ & $\begin{array}{l}0 \\
6\end{array}$ & $\begin{array}{r}0 \\
20\end{array}$ & $\begin{array}{r}0 \\
16\end{array}$ & $\begin{array}{l}0 \\
0\end{array}$ & $\begin{array}{r}0 \\
14\end{array}$ & $\begin{array}{l}0 \\
0\end{array}$ & $\begin{array}{l}0 \\
4\end{array}$ \\
\hline 10 & $\begin{array}{c}\text { Sexual } \\
\text { Asexual }\end{array}$ & $\begin{array}{l}0 \\
0\end{array}$ & $\begin{array}{l}0 \\
0\end{array}$ & $\begin{array}{r}0 \\
20\end{array}$ & $\begin{array}{r}0 \\
15\end{array}$ & $\begin{array}{l}0 \\
0\end{array}$ & $\begin{array}{l}0 \\
0\end{array}$ & $\begin{array}{l}0 \\
0\end{array}$ & $\begin{array}{l}1 \\
5\end{array}$ \\
\hline 11 & $\begin{array}{c}\text { Sexual } \\
\text { Asexual }\end{array}$ & $\begin{array}{l}0 \\
5\end{array}$ & $\begin{array}{l}0 \\
2\end{array}$ & $\begin{array}{r}0 \\
20\end{array}$ & $\begin{array}{r}0 \\
17\end{array}$ & $\begin{array}{l}0 \\
0\end{array}$ & $\begin{array}{r}0 \\
18\end{array}$ & $\begin{array}{l}0 \\
0\end{array}$ & $\begin{array}{l}0 \\
3\end{array}$ \\
\hline 12 & $\begin{array}{l}\text { Sexual } \\
\text { Asexual }\end{array}$ & $\begin{array}{r}0 \\
10\end{array}$ & $\begin{array}{l}0 \\
6\end{array}$ & $\begin{array}{r}0 \\
20\end{array}$ & $\begin{array}{r}0 \\
20\end{array}$ & $\begin{array}{l}0 \\
0\end{array}$ & $\begin{array}{r}0 \\
14\end{array}$ & $\begin{array}{l}0 \\
0\end{array}$ & $\begin{array}{l}0 \\
0\end{array}$ \\
\hline 13 & $\begin{array}{c}\text { Sexual } \\
\text { Asexual }\end{array}$ & & $\begin{array}{l}0 \\
8\end{array}$ & & $\begin{array}{r}0 \\
18\end{array}$ & & $\begin{array}{r}0 \\
12\end{array}$ & & $\begin{array}{l}0 \\
2\end{array}$ \\
\hline 14 & $\begin{array}{c}\text { Sexual } \\
\text { Asexual }\end{array}$ & & $\begin{array}{l}0 \\
9\end{array}$ & & $\begin{array}{r}0 \\
13\end{array}$ & & $\begin{array}{r}0 \\
11\end{array}$ & & $\begin{array}{l}4^{* *} \\
7\end{array}$ \\
\hline 15 & $\begin{array}{c}\text { Sexual } \\
\text { Asexual }\end{array}$ & & $\begin{array}{r}0 \\
15\end{array}$ & & $\begin{array}{r}0 \\
14\end{array}$ & & $\begin{array}{l}1 \\
5\end{array}$ & & $\begin{array}{l}1 \\
6\end{array}$ \\
\hline 16 & $\begin{array}{c}\text { Sexual } \\
\text { Asexual }\end{array}$ & & $\begin{array}{r}0 \\
18\end{array}$ & & $\begin{array}{r}0 \\
11\end{array}$ & & $\begin{array}{l}2^{* * *} \\
2\end{array}$ & & $\begin{array}{l}0 \\
8\end{array}$ \\
\hline 17 & $\begin{array}{c}\text { Sexual } \\
\text { Asexual }\end{array}$ & & $\begin{array}{r}0 \\
17\end{array}$ & & $\begin{array}{r}0 \\
14\end{array}$ & & $\begin{array}{l}0 \\
3\end{array}$ & & $\begin{array}{l}1 \\
6\end{array}$ \\
\hline 18 & $\begin{array}{c}\text { Sexual } \\
\text { Asexual }\end{array}$ & & $\begin{array}{r}0 \\
10\end{array}$ & & $\begin{array}{r}0 \\
11\end{array}$ & & $\begin{array}{r}0 \\
10\end{array}$ & & $\begin{array}{l}0 \\
9\end{array}$ \\
\hline 19 & $\begin{array}{l}\text { Sexual } \\
\text { Asexual }\end{array}$ & & $\begin{array}{r}0 \\
12\end{array}$ & & $\begin{array}{r}0 \\
13\end{array}$ & & $\begin{array}{l}4^{*} \\
8\end{array}$ & & $\begin{array}{l}0 \\
7\end{array}$ \\
\hline 20 & $\begin{array}{l}\text { Sexual } \\
\text { Asexual }\end{array}$ & & $\begin{array}{r}1 \\
14\end{array}$ & & $\begin{array}{r}0 \\
36\end{array}$ & & $\begin{array}{l}24^{* * * * *} \\
6\end{array}$ & & $\begin{array}{r}0 \\
21\end{array}$ \\
\hline 21 & $\begin{array}{l}\text { Sexual } \\
\text { Asexual }\end{array}$ & & $\begin{array}{r}0 \\
15\end{array}$ & & & & $\begin{array}{l}8^{* * * * *} \\
5\end{array}$ & & \\
\hline 22 & $\begin{array}{c}\text { Sexual } \\
\text { Asexual }\end{array}$ & & $\begin{array}{r}0 \\
16\end{array}$ & & & & $\begin{array}{l}4^{* * * *} \\
4\end{array}$ & & \\
\hline 23 & $\begin{array}{c}\text { Sexual } \\
\text { Asexual }\end{array}$ & & $\begin{array}{r}0 \\
14\end{array}$ & & & & $\begin{array}{l}0 \\
6\end{array}$ & & \\
\hline
\end{tabular}


TABLE 3 (cont.)

\begin{tabular}{|c|c|c|c|}
\hline Week & Type & $\begin{array}{l}\text { Genotype C } \\
\text { Experiments }\end{array}$ & \\
\hline 24 & $\begin{array}{l}\text { Sexual } \\
\text { Asexual }\end{array}$ & $\begin{array}{r}6 \\
0 \\
11\end{array}$ & $\begin{array}{l}6 \\
1 \\
9\end{array}$ \\
\hline 25 & $\begin{array}{l}\text { Sexual } \\
\text { Asexual }\end{array}$ & $\begin{array}{r}0 \\
27\end{array}$ & $\begin{array}{l}4^{* * *} \\
13\end{array}$ \\
\hline 26 & $\begin{array}{c}\text { Sexual } \\
\text { Asexual }\end{array}$ & $\begin{array}{r}0 \\
23\end{array}$ & $\begin{array}{l}0 \\
7\end{array}$ \\
\hline
\end{tabular}

The data can still, however, be analysed in order to test for frequencydependent selection. If selection is constant, then in the appropriate haploid model for gene frequency change, a graph of $\ln (q / 1-q)$ against time should have a constant gradient (where $q$ is the frequency of one clone). If the slope of such a line decreases with increasing frequency of the favoured clone, this represents stabilising frequency-dependent selection even if one clone is fixed by artificial selection.

Thus for each week the selection, $S$, was calculated as $\ln \left(q_{0}(1-\right.$ $\left.\left.q_{1}\right) / q_{1}\left(1-q_{0}\right)\right)$ where $q_{0}=$ the frequency in week 0 , and $q_{1}=$ the frequency in week 1 , using the data from experiments 1-4 and from experiments 5-8. Regressions were performed of $S$ against $\left(q_{0}+q_{1}\right) / 2$. These should give positive slopes if there is stabilising frequency-dependent selection. For experiments 1-4, with clones B and C, separate regressions were performed of $S$ on $q$ for each experiment. The lines produced were homogeneous (as a t-test showed no significant variation in their slopes, and a Hartley's F-max test showed no significant variation in their residual variances). The pooled data from these experiments gave a regression of $S=-0 \cdot 31-0 \cdot 13 q$. The residuals showed no significant departure from normality in a ShapiroWilks W test and the slope was not significantly different from zero in a t-test.

For experiments 5-8, with clones $\mathrm{A}$ and $\mathrm{C}$, separate regression slopes were calculated for $S$ on $q$. t-tests showed none of these to be significantly different from any other, but a Hartley's F-max test showed heterogeneity in the residual variances at the 5 per cent level. This heterogeneity was eliminated by pooling only the results from experiments 5, 6 and 8 (giving a line of $S=-0.39+0.47 q$, which was not significantly different from a slope with zero gradient or from the slope in experiment 7 ), or by pooling only experiments 5,7 and 8 (giving a line of $S=-1 \cdot 06+1 \cdot 28 q$, which was not significantly different from a slope of zero or from that of experiment 6 ). The total pooled data gave a line of $S=-0 \cdot 37+0.41 q$, which was a slope not significantly different from zero, and which gave residuals not significantly non-normal in a Shapiro-Wilks W test. In experiments 9 and 10 not enough data were available for statistical analyses to be performed.

In experiments 11-18 clone $\mathrm{G}$ was used. This clone possessed a mutation (probably recessive) causing a lack of pigment in the compound eye. It was kindly supplied by Dr Elaine Watts and Dr Steven Young of Imperial College Field Station at Ashurst Lodge, University of London. The visible difference between clones allowed the genotypes of the whole population to be assessed. Figures 4-6 show the changes in the frequencies of blackeyed clones with clone $\mathrm{G}$ when the black-eyed clone was $\mathrm{C}$ (experiments 


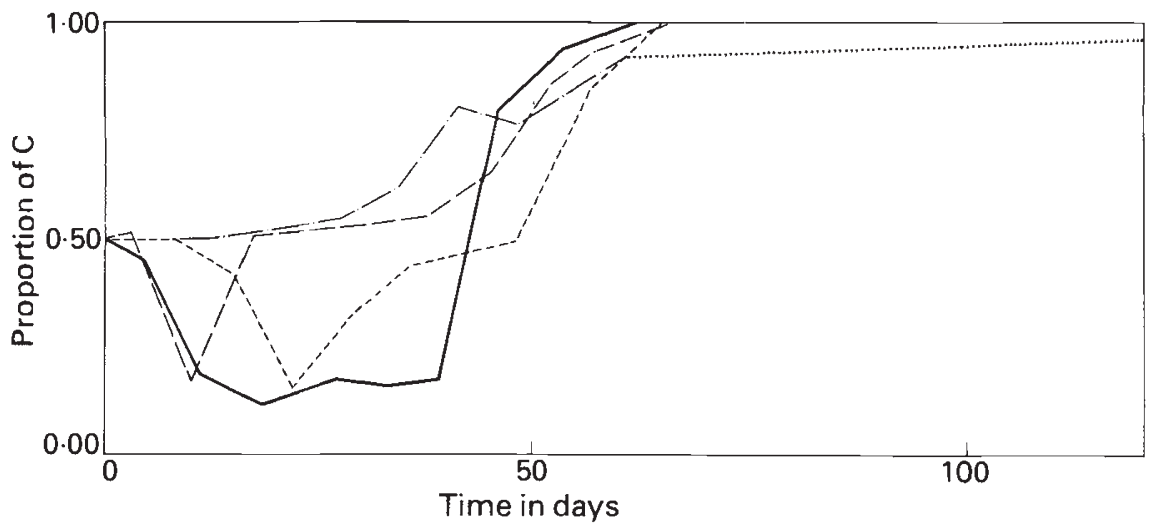

FIG. 4.-Variations in the proportions of clone $C$ in the whole population in competition with clone $G$ in experiments $11-14$. Clone $C$ comprised 50 per cent of the population initially in each experiment. $=$ Experiment $11,---=$ Experiment $12,-\cdots=$ Experiment $13, \cdot-\cdots-=$ Experiment $14, \cdots \cdots=$ Data unavailable.

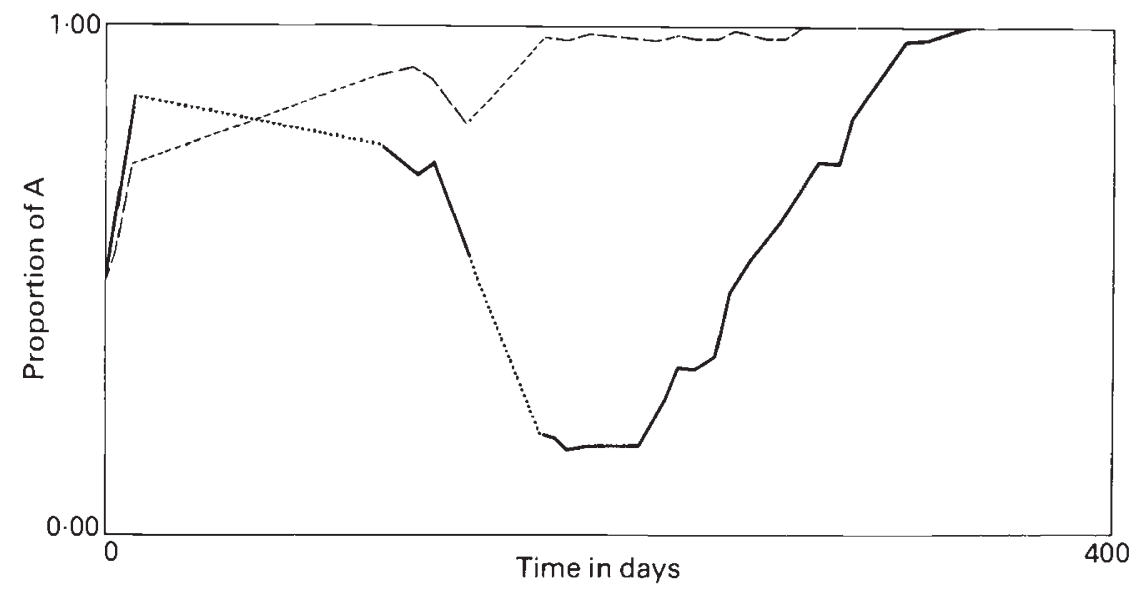

FIG. 5.-Variation in the proportions of clone $A$ in the whole population, in competition with clone $\mathrm{G}$ in experiments 15 and 16 . Clone $A$ comprised 50 per cent of the population initially in each experiment. $-=$ Experiment $15,----=$ Experiment $16, \cdots \cdots=$ Data unavailable.

11-14), A (experiments 15 and 16), and F (experiment 17). These figures show the frequencies based on the whole population but frequencies based on adults were also obtained. A further experiment with clone A (experiment 18 ) was started but accidentally contaminated with clone $\mathrm{C}$ after a period of 21 days.

As above, regressions can be done of $S$, defined as $1 / t \ln \left(q_{0}(1-\right.$ $\left.q_{1}\right) / q_{1}\left(1-q_{0}\right)$ ) where $t$ is the time in weeks separating the two clone frequency measurements $q_{0}$ and $q_{1}$, against $q_{0}$ (which is used in this case as it is a population frequency, as opposed to the sample frequencies used in experiments 1-10 which would give spurious evidence of frequencydependent selection if they were used instead of $\left(q_{0}+q_{1}\right) / 2$ as the independent variable). Two separate regressions can be done for each experiment, 


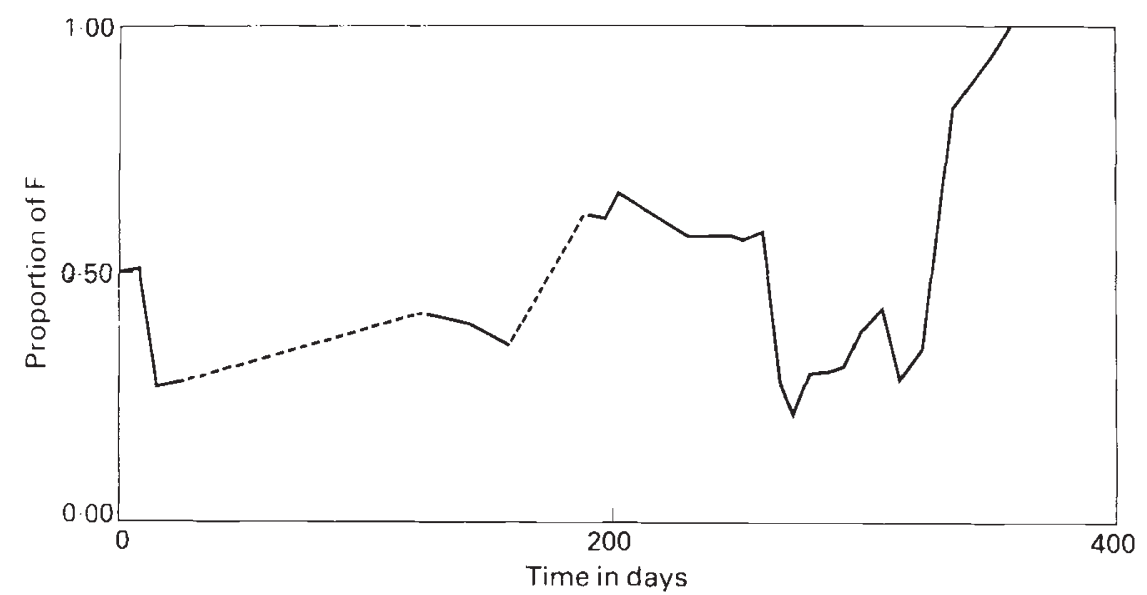

FIG. 6.-Variation in the proportion of clone $\mathrm{F}$ in the whole population in competition with clone $\mathrm{G}$ in experiment 17 , in which both clones initially had equal frequencies.

one based on $q$ values in the whole population and the other based on $q$ values among adults.

For experiments 11-14, the regressions of $S$ on $q$ for the adult data showed no differences in their slopes, but were heterogeneous at the 5 per cent level in a Hartley's F-max test. The heterogeneity could be eliminated by pooling only the results from experiments 12,13 and 14 (giving a line of $S=0 \cdot 30-1 \cdot 14 q$, which was not significantly different from a slope of zero gradient or from the slope of experiment 11 ), or by pooling only experiments 11,12 , and 14 (giving a line of $S=0.61-1.47 q$, which was not significantly different from a slope of zero gradient or from the slope of experiment 13). However, the slope of experiment $11,6 \cdot 58$, but not that of any other experiment, was significantly greater than zero at the 5 per cent level.

The regression of $S$ on $q$ for the whole population in these experiments were all homogeneous in their slopes and residual variances, and no slope was significantly different from zero. Pooling all experiments gave a line of $S=0.08-0.52 q$, which was not significantly different from slope of zero. In none of these regressions were the residuals significantly non-normal.

For experiments 11-18 the number of parthenogenetic eggs carried by each adult female was counted, and the mean egg number for each clone, $R$, and the difference between the $R$ values of the black-eyed clone and clone $\mathrm{G}$ at any time was calculated. This quantity was called $D$ and it was regressed against the frequency of the black-eyed clone. Stabilising frequency-dependent selection acting through fertility differences would result in a negative gradient.

For clones $A$ and $\mathrm{G}$ in experiments $11-14$, individual regressions of $D$ on $q$ for the adult data gave non-significant slopes which showed no significant heterogeneity, but the residual variances of the regressions were heterogeneous. This heterogeneity could be eliminated by pooling 11 and 13 (giving a line of $D=0 \cdot 23-0 \cdot 38 q$ ) and 12 and 14 (giving a line of $D=-0 \cdot 08+0 \cdot 27 q)$. Neither of these slopes was significantly different from zero and neither were they significantly different from each other. Individual regressions of $D$ on $q$ for all the data in experiments 11-14 gave non- 
significant slopes which showed no significant heterogeneity, but again the residual variances were heterogeneous. This heterogeneity could be eliminated by pooling 11 and 13 (giving a line of $D=-0.77+1 \cdot 02 q$ ) and 12 and 14 (giving a line of $D=-0 \cdot 15+0 \cdot 41 q$ ). Neither of these slopes was significantly different from zero, and neither were they significantly different from each other.

If the variation in the values of $D$ obtained is of selective importance, then regressions of $S$ on the $D$ value in the initial population used to calculate $S$ will give a negative slope. Individual regression of $S$ on $D$ for experiments 11-14 using the data for adults gave non-significant slopes which showed no significant heterogeneity either in their slopes or in their residual variances. Pooling the experiments gave the line $S=0 \cdot 32-0 \cdot 12 D$, which was not significantly different from a slope of zero, but which left residuals which were significantly non-normal at the 1 per cent level. To eliminate this, the values for $S$ and $D$ were transformed using an Arctan transformation and a new regression performed. For the individual experiments all slopes were again non-significant and there was no heterogeneity between experiments in their slopes or in their residual variances. The pooled regression gave $\operatorname{Arctan} S=-0.17-0.11$ Arctan $D$, which was a non-significant slope, and now the residuals were not significantly nonnormal.

Individual regressions of untransformed values of $S$ (based on all the data) on $D$ for experiments 11-14 gave non-significant slopes, and there was no heterogeneity between experiments. The pooled data gave a line of $S=0 \cdot 49-0 \cdot 28 D$, which was not significantly different from a slope of zero, but again the residuals were non-normal. In this case such non-normality remained when an Arctan transformation was applied to the data.

For experiments 15 and 16, regressions of $S$ on $q$, using either the data based on adults, or that based on the whole population, gave non-significant slopes, which were homogeneous, and no heterogeneity was found in the residual variances. Pooling these experiments gave a line of $S=$ $0 \cdot 46+0 \cdot 42 q$, when data were based on the adults, and $S=-0 \cdot 28+0 \cdot 16 q$, when data were based on the whole population. Neither slope was significantly different from zero. All the regressions gave residuals which failed to deviate significantly from normality.

Regressions of $D$ on $q$ for experiments 15 and 16 using the data from adults gave slopes which were significantly different at the 5 per cent level, and which had residual variances which were significantly different at the $0 \cdot 1$ per cent level. The slope from experiment $16,-7 \cdot 59$, was different from zero at the 0.005 per cent level. However, the residuals from both regressions were significantly non-normal at the 1 per cent level. Pooling the experiments gave a line of $D=0.98-0.79 q$, which is a slope not significantly different from zero, but which, again, gave residuals which were significantly non-normal at the 1 per cent level. An Arctan transformation on the values for $D$ failed to remove the non-normality of the residuals in any of the regressions. Regressions of $D$ on $q$ for experiments 15 and 16 using data from the whole population gave no significant difference in slopes but a difference in residual variances at the 1 per cent level. The slope in $16,-8 \cdot 43$, was different from zero at the 1 per cent level. Both regressions gave residuals which were significantly non-normal 
at the 1 per cent level. Pooling the experiments gave a line of $D=$ $0 \cdot 25+0 \cdot 24 q$, which was a slope not significantly different from zero, but which has residuals which were significantly non-normal at the 1 per cent level. Arctan transformations on the $D$ values failed to remove the nonnormality from the residuals for any regression.

Regressions of $S$ based on adults on $D$ for these two experiments gave lines which had non-significant slopes, and which were homogeneous with respect to their slopes and their residual variances. Pooling them gave a line of $S=-0 \cdot 16-0 \cdot 04 D$ which was not significantly different from zero. Similar homogeneity and non-significance was found for the regressions of $S$, based on the whole population in these experiments, against $D$, the pooled regression being $S=-0.13-0.03 D$, which was again non-significant.

In experiment 17 the regression of $S$ on $q$ for data based on adults gave a line of $S=0.30+0.55 q$, with no deviation from normality in the residuals. The slope is not significantly different from zero. Data from the whole population gave $S=-0 \cdot 16+0 \cdot 42 q$, which was a non-significant slope, but in this case the residuals were significantly non-normal at the 2 per cent level, a result that remained true when an Arctan transformation was applied to the values of $S$. Regressions of $D$ on $q$ for experiment 17, using data based on adults, and data based on the whole population, gave lines of $D=0.12-0.54 q$, and $D=-0.12+0.03 q$ respectively. Each slope was not significantly different from zero, but had residuals which were significantly non-normal at the 1 per cent level, a result that remained true when an Arctan transformation was applied to the values of $D$.

The regression of $S$ on $D$ for data from adults in this experiment gave a line of $S=-0.07-0.14 D$, with no evidence of non-normality in the residuals. This slope was not significantly different from zero. Using data from the whole population, the regression of $S$ on $D$ gave a line of $S=-0.04-0.31 D$, which was significantly different from zero at the 0.5 per cent level, but which gave residuals which were significantly non-normal at the 1 per cent level. An Arctan transformation gave Arctan $S=$ $-0.03-0.45 \operatorname{Arctan} D$, which again had a slope significantly different from zero at the 0.5 per cent level, but which now had residuals which were not significantly non-normal.

Thus, in summary, there were only two cases where the regression slope for any experiment was both significantly different from zero and statistically legitimate (as is shown by the residuals being normally distributed). In experiment $11, S$ increases with $q$ when the adult data are studied, the level of significance being 5 per cent, and in experiment 17, the Arctantransformed $S$ values decrease with increasing $\operatorname{Arctan} D$, when data from the whole population are studied. In this second case the level of significance is 0.5 per cent. In a series of regressions of this kind, a single significance value of 5 per cent is likely to be produced by chance, but one of 0.5 per cent is unlikely. It can be concluded that there is no real evidence that either selection or the difference in fecundity between clones varies with clone frequency, but there is some evidence that differences in fecundity between clones result in selective differences.

A crude analysis of the species diversity of the culture in experiment 5 revealed 8 coexisting Protozoan genera (five of which were photosynthetic), 3 rotifer genera and 3 other crustacean general. 


\section{Discussion}

One danger in analyses of this type is that of making a Type 2 statistical error, accepting an inadequately tested null hypothesis (Dixon and Massey, 1969). While the slopes of the regressions are almost all non-significant, in many cases they are very different from zero. However, in all the data taken together, there seems to be no general trend towards slopes showing stability. The results of experiments 6 and 8 , where in each case clone $C$ rises from an initial frequency of 10 per cent to an approximately stable frequency of 60 to 80 per cent, are suggestive of a stable equilibrium, but in the absence of further data no conclusion is possible.

Most demonstrations of frequency-dependent selection have shown the phenomenon at high density. Figure 7 shows the variation in population

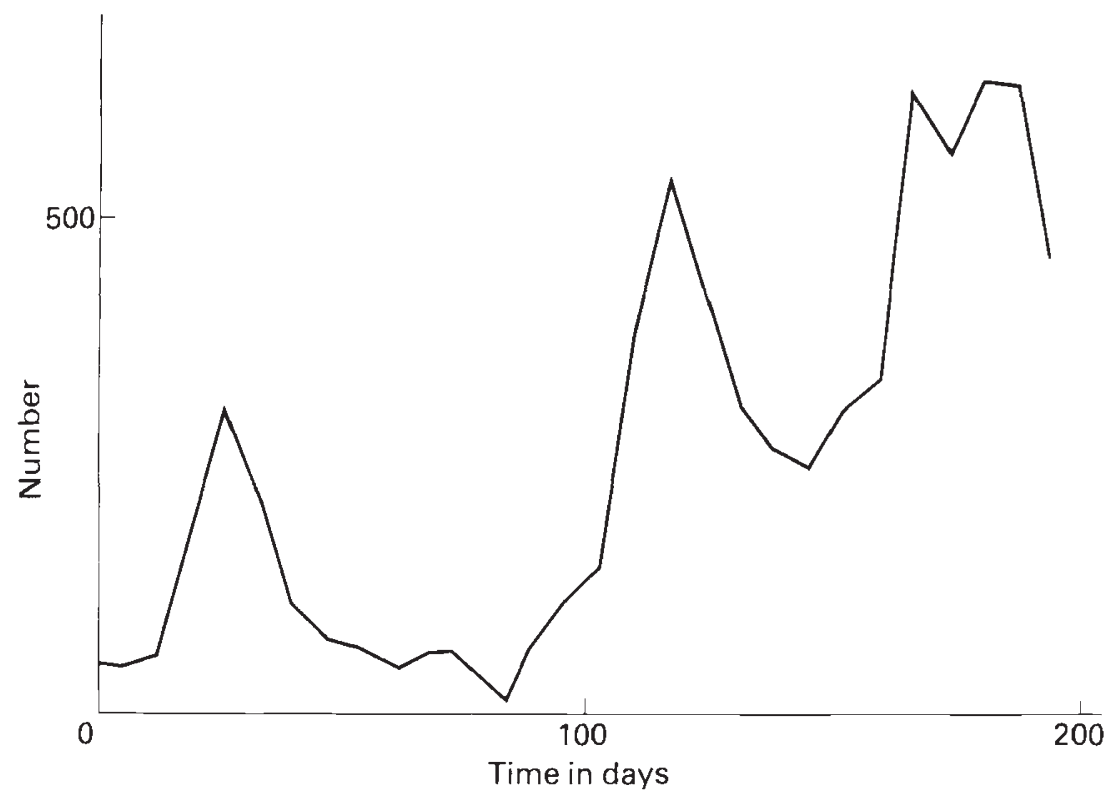

FIG. 7.-The number of individuals against time in days in experiment 5 .

size with time in experiment 5. This pattern of fluctuation was typical of the experiments. The populations were at or near the maximum population size for only a small proportion of the time. To restrict the analysis to high density data would reduce the amount of data available below that on which regressions could legitimately be performed. However, while the populations showed consistent fluctuations in size there is no evidence that food was not limiting throughout. Populations of Daphnia species with controlled food supplies still show oscillations in density. This is true of D. pulex (Frank et al., 1957; Frank, 1960), D. obtusa (Slobodkin, 1954) and D. magna (Pratt, 1943; Smith, 1963). Both the size and frequency of these oscillations are predicted by the model of Frank (1960), which differs from the logistic model of population growth in that it makes the 
density-dependent effect on egg-production dependent upon the density five days prior to laying. It seems likely that almost all of the time the populations studied above were limited by food.

Thus there is no evidence for frequency-dependent selection acting between clones in a moderately complex environment. Such selection would be expected to act between clones if most allelic differences are acted on by stabilising frequency-dependent selection, as the clones will differ at many genetic loci. The vast majority of the data presented here are from experiments where the clones are derived from different sources. In experiments 9 and 10, where the clones were derived from the same source at the same time, the process of fixation was so rapid that not enough data were produced for a regression analysis. In a similar experiment, Snell (1979) failed to find stable coexistence of clones of rotifers (Asplanchna species). Kosuda (1981), however, studying the replacement of a $\mathrm{lac}^{-}$with a lac ${ }^{+}$strain in Escherichia coli, found stabilising frequencydependent selection in a curvilinearity in a plot of $\ln (q / 1-q)$ against time, where $q$ is the frequency of the $l_{a c}^{-}$strain.

The level of genetic diversity observed in wild populations by enzyme electrophoresis is higher than that predicted by the "classical" theory of population genetics (Lewontin, 1974). Considerable effort has been exerted in the search for systems of natural selection such as heterozygote advantage and stabilising frequency-dependent selection which can maintain polymorphisms. However, the discovery of the selection systems maintaining polymorphisms does not elucidate the forces determining what proportion of the geriome will be subject to such selection. The analogy of Clarke (1979) between the maintenance of polymorphism by frequency-dependent selection and the maintenance of diverse species communities by density-dependent effects is revealing and accurate. Predictions of the level of polymorphism cannot be derived from demonstrations of frequencydependent selection any more than predictions of community diversity can be derived from demonstrations that individual species have stable densities. Furthermore, as selectively maintained polymorphisms will accumulate in wild populations, the finding of a particular level of polymorphism, even if it is higher than expected, does not require for its explanation an a priori expectation of selection acting on randomly chosen alleles. Nevertheless, the lack of experimental evidence reported here for frequency-dependent selection fails to agree with the genetic diversity observed in wild populations of Cladocera, which in many cases appears to be selectively important (Hebert, 1972, 1974a, 1974b; Hebert and Ward, 1976; Hebert et al., 1972; Young, 1975, 1979a, 1979b) for not only is diversity not easily explained by heterozygote advantage, it cannot (in view of the rarity of sexuality) be due to frequency-dependent mating success.

Acknowledgements.-I would like to thank Dr I. E. Lush for supervision during the course of this work, Dr E. Watts and Dr S. Young for kindly supplying me with individuals of the white-eyed clone G, Dr R. I. Gilbert for statistical advice, Dr B. Charlesworth, Dr J. S. Jones and Mr R. F. Probert for advice. This work was carried out while I was in receipt of a Junior Research Fellowship at the Royal Free Hospital School of Medicine, University of London. 


\section{REFERENCES}

ALLEN, J. A. 1972. Evidence for stabilising and apostatic selection by wild blackbirds. Nature, 237, 348-349.

ALLEN, J. A., AND CLARKE, B. C. 1968. Evidence for apostatic selection by wild passerines. Nature, 220, $501-502$.

ANDERSON, B. G., AND JENKINS, J. C. 1942. A time study of the events in the life span of Daphnia magna. Biol. Bull., 83, 260-272.

ANGUS, R. A. 1978. Daphnia and the search for heterosis. Amer. Natur., 112, 955-956.

AYAla, F. J., AND CAMPBELl, C. A. 1974. Frequency dependent selection. Ann. Rev. Ecol. Syst., 5, 115-138.

BANTA, A. M., WOOD, T. R., BROWN, L. A., AND INGLE, L. 1939. Studies on the physiology, genetics and evolution of some Cladocera. Carnegie Institution Publication. Number, 39.

BERENEK, A. P. 1974. Stable and nonstable resistance to dimethoate in the peach-potato aphid (Myzus persicae). Entomol. Exp. Appl., 17, 381-390.

BERGER, E. 1976. Heterosis and the maintenance of enzyme polymorphism. Amer. Natur., $110,823-839$.

BERGER, E., AND SUTHERLAND, J. 1978. Allozyme variation in two natural populations of Daphnia pulex. Heredity, 41, 13-24.

BIRLEY, A. J., AND BEARDMORE, J. A. 1977. Genetical composition, temperature, density and selection in an enzyme polymorphism. Heredity, 39, 133-144.

BROWER, L. P., COOK, L. M., AND CROZE, H. J. 1967. Predator responses to artificial Batesian mimics released in a neotropical environment. Evolution, 21, 11-23.

BUNTING, S., AND VAN EMDEN, H. F. 1980. Rapid response to selection for increased esterase activity on small populations of an apomictic clone of Myzus persicae. Nature, $285,502-503$.

CLARKE, B. C. 1962. Balanced polymorphism and the diversity of sympatric species. Systematics Association Publications, 4, 47-70.

CLARKE, B. C. 1975. Frequency-dependent and density-dependent natural selection. In The Role of Natural Selection In Human Evolution, ed. F. M. Salzano, pp. 187-200. Amsterdam, North-Holland.

CLARKE, B. C. 1976. The ecological genetics of host-parasite relationships. In Genetic aspects of host-parasite relationships, eds. A. E. R. Taylor and R. Muller, pp. 87-103. Oxford, Blackwell.

CLARKe, B. C. 1979. The evolution of genetic diversity. Proc. R. Soc. Lond. B., 205, 453-474.

CLARKE, B. C., AND ALLENDORF, F. W. 1979. Frequency-dependent selection due to kinetic differences between allozymes. Nature, 279, 732-734.

DANFORD, N. D., AND BEARDMORE, J. A. 1979. Biochemical properties of esterase-6 in Drosophila melanogaster. Biochem. Genet., 17, 1-22.

DAY, T. H., HILLIER, P. C., AND CLARKE, B. C. 1974. Properties of genetically polymorphic isozymes of alcohol dehydrogenase in Drosophila melanogaster. Biochem. Genet., 11, 141-153.

DEMPSTER, E. R. 1955. Maintenance of genetic heterogeneity. Cold Spring Harbor Symp. Quant. Biol., 20, 25-32.

DIXON, W. J., AND MASSEY, F. J., JR. 1969. Introduction to statistical analysis. 3rd Edition. McGraw-Hill, New York.

DOLAN, R., AND ROBERTSON, A. 1975. The effect of conditioning the medium in Drosophila, in relation to frequency-dependent selection. Heredity, 35, 311-316.

EHRMAN, L. 1966. Mating success and genotype frequency in Drosophila. Animal Behaviour, 14, 332-339.

EHRMAN, L., SPASKY, B., PAVLOVSKY, O., AND DOBZHANSKY, TH. 1965. Sexual selection, geotaxis and chromosomal polymorphism in experimental populations of Drosophila pseudoobscura. Evolution, 19, 337-346.

FRANK, P. W. 1960. Prediction of population growth form in Daphnia pulex cultures. Am. Nat., 94, 357-372.

FRANK, P. W., BOLL, C. D., AND KELLY, R. W. 1957. Vital statistics of laboratory cultures of Daphnia pulex Degeer as related to density. Physiol. Zool., 30, 287-305.

GIBSON, J. 1970. Enzyme flexibility in Drosophila melanogaster. Nature, 227, 959-960.

GREEN, J. 1954. Size and reproduction in Daphnia magna. Proc. Zool. Soc. London, 124, 535-545.

HARRIS, H. 1971. Protein polymorphism in man. Can. J. Genet. Cytol., 13, 381-396. 
HEBERT, P. D. N. 1972. Enzyme variability in natural populations of Daphnia magna. Ph.D. Thesis, University of Cambridge.

HEBERT, P. D. N. 1974a. Ecological differences between genotypes in a natural population of Daphnia magna. Heredity, 33, 327-337.

HEBERT, P. D. N. 1974 b. Enzyme variability in natural populations of Daphnia magna. II. Genotypic frequencies in permanent populations. Genetics, 77, 323-334.

HEBERT, P. D. N. 1974c. Enzyme variability in natural populations of Daphnia magna. III. Genotypic frequencies in temporary populations. Genetics, 77, 335-341.

HEBERT, P. D. N. 1975. Enzyme variability in natural populations of Daphnia magna. I. Population structure in East Anglia. Evolution, 28, 546-556.

HEBERT, P. D. N. 1978. The population biology of Daphnia (Crustacea: Daphnidae). Biol. Rev., 53, 387-436.

HEBERT, P. D. N., AND CREASE, T. J. 1980. Clonal coexistence in Daphnia pulex (Leydig): Another planktonic paradox. Science, 207, 1363-1365.

HEBERT, P. D. N., AND WARD, R. D. 1972. Inheritance during parthenogenesis in Daphnia magna. Genetics, 71, 639-642.

HEBERT, P. D. N., AND WARD, R. D. 1976. Enzyme variability in natural populations of Daphnia magna. IV. Ecological differentiation and frequency changes of genotypes at Audley End. Heredity, 36, 331-341.

HEBERT, P. D. N., WARD, R. D., AND GIBSON, J. B. 1972. Natural selection for enzyme variants among parthenogenetic Daphnia magna. Genet. Res., 19, 173-176.

HEDRICK, P. W., GINEVAN, M. E., AND EWING, E. P. 1976. Genetic polymorphism in heterogeneous environments. Ann. Rev. Ecol. Syst., 7, 1-33.

HOEKSTRA, R. F. 1978. Sufficient conditions for polymorphism with cyclical selection in a subdivided population. Genet. Res., 31, 67-73.

HUANG, S. L,, SINGH, M., AND KOJIMA, K. I. 1971. A study of frequency-dependent selection observed on the esterase- 6 locus of Drosophila melanogaster using a conditioned media method. Genetics, 68, 97-114.

KOEHN, R. K. 1969. Esterase heterogeneity: dynamics of a polymorphism. Science, 163, 943-944.

KOJIMA, K. I., AND HUANG, S. 1972. Effects of population density on the frequencydependent selection in the esterase- 6 locus of Drosophila melanogaster. Evolution, 26, 313-321.

KOJIMA, K. I., AND TOBARI, Y. N. $1969 a$. The pattern of viability changes associated with genotype frequency at the alcohol dehydrogenase locus in a population of Drosophila melanogaster. Genetics, 61, 201-209.

KOJIMA, K. I., AND TOBARI, Y. N. 1969b. Selective modes associated with karyotypes in Drosophila ananassea. II. Heterosis and frequency-dependent selection. Genetics, 63, 639-651.

KOJIMA, K. I., AND YARBOROUGH, K. 1967. Frequency-dependent selection at the esterase6 locus of Drosophila melanogaster. Proc. Nat. Acad. Sci. U.S.A., 57, 645-649.

KOSUDA, KAZUHIKO. 1981. On the mode of selection in Escherichia coli. Genetica, 55, 17-21.

KRISTJANSSON, F. K. 1963. Genetic control of two pre-albumins in pigs. Genetics, 48, 1059-1063.

LEVENE, H. 1953. Genetic equilibrium when more than one niche is available. Amer. Natur., $87,331-333$.

LEWONTIN, R. C. 1974. The genetic basis of evolutionary change. Columbia University Press, New York.

MANNING, B. J., KERFOOT, W. C., AND BERGER, E. M. 1978. Phenotypes and genotypes in Cladoceran populations. Evolution, 32, 365-374.

MARINKOVIC, D., AND AYALA, F. J. 1975. Fitness of allozyme variants in Drosophila pseudoobscura. I. Selection at the Pgm-1 and Me-2 loci. Genetics, 79, 85-95.

MAYNARD SMITH, J., AND HOEKSTRA, R. 1980. Polymorphism in a varied environment: how robust are the models? Genet. Res., 35, 45-58.

MORGAN, P. 1976. Frequency-dependent selection at two enzyme loci in Drosophila melanogaster. Nature, 263, 765-766.

NARISE, S. 1971. Biochemical differences between the $\alpha$-and $\beta$-esterase isozymes in Drosophila virilis. Drosophila Information Service, 46, 115.

NARISE, S. 1973. Esterase isozymes of Drosophila virilis: purification and properties of $\alpha$ and $\beta$-esterase isozymes. Japan J. Genetics, 48, 119-132.

NASSAR, R. 1979. Frequency-dependent selection at the LAP locus in Drosophila melanogaster. Genetics, 91, 327-338. 
NASSAR, R. F., MUHS, H. S., AND COOK, R. D. 1973. Frequency-dependent selection at the Payne inversion in Drosophila melanogaster. Evolution, 27, 558-564.

OBRESHKOVE, V., AND FRASER, A. W. 1940. Growth and differentiation of Daphnia magna eggs in vitro. Biol. Bull., 78, 428-436.

PRATT, D. M. 1943. Analysis of population development of Daphnia at different temperatures. Biol. Bull., 85, 116-140.

PROUT, T. 1968. Sufficient conditions for a multiple niche polymorphism. Amer. Natur., $102,493-496$.

Slobodkin, L. B. 1954. Population dynamics of Daphnia obtusa. Kutz. Ecol. Monogr., 24, 69-88.

SMITH, F. E. 1963. Population dynamics of Daphnia magna and a new model for population growth. Ecology, 44, 651-663.

SMITH, M. Y., AND FRASER, A. 1976. Polymorphism in a cyclic parthenogenetic species Simocephalus serrulatus. Genetics, 84, 631-637.

SNELL, T. 1979. Interspecific competition and population structure in rotifers. Ecology, 60 , 494-502.

SNYDER, T. P., AND A YALA, F. J. 1979. Frequency-dependent selection at the PGM-1 locus in Drosophila pseudoobscura. Genetics, 92, 995-1003.

SPIESS, E. B. 1957. Relation between frequencies and adaptive values of chromosomal arrangements in Drosophila persimilis. Evolution, 11, 84-93.

SPIESS, F. B. 1970. Mating propensity and its genetic basis in Drosophila. In Essays in Evolution and Genetics, eds. M. K. Hecht and W. C. Steere, pp. 315-379. Appleton, New York.

STEWART, F. M., AND LEVIN, B, R. 1973. Partitioning of resources and the outcome of interspecific competition: a model and some general considerations. Amer. Natur., 107, 171-198.

SUOMALAINEN, E., SAURA, A., LOKKI, J., AND TEERI, T. 1980. Absence of variation within parthenogenetic aphid clones. Theor. Appl. Genet., 57, 129-132.

TOBARI, Y. N., AND KOJIMA, K. I. 1967. Selective modes associated with inversion karyotypes in Drosophila ananassea. Frequency-dependent selection. Genetics, 57, 179-188.

WAllaCE, B. 1968. Polymorphism, population size and genetic load. In Population Biology and Evolution. ed. R. C. Lewontin, pp. 87-108, University Press, Syracuse.

WARD, R. D. 1974. Alchohol dehydrogenase in Drosophila melanogaster: activity variation in natural populations. Biochem. Genet., 12, 449-458.

woOD, T. R. 1932. Resting eggs that fail to rest. Amer. Natur., 77, 277-281.

WRIGHT, S., AND DOBZHANSKY, TH. 1946. Genetics of natural populations. XII. Experimental reproduction of some of the changes caused by natural selection in certain populations of Drosophila pseudoobscura. Genetics, 31, 125-156.

YAMAZAKI, T, 1971. Measurement of fitness at the esterase-5 locus in Drosophila pseudoobscura. Genetics, 67, 579-603.

YOSHIMARU, H., AND MUKAI, T. 1979, Lack of experimental evidence for frequencydependent selection at the alcohol dehydrogenase locus in Drosophila melanogaster. Proc. Nat. Acad. Sci. U.S.A., 76, 876-878.

YOUNG, P. W. 1975. Enzyme polymorphisms and reproduction in Daphnia magna. Ph.D. Thesis. University of Cambridge.

YOUNG, J. P. W. 1979a. Enzyme polymorphism and cyclic parthenogenesis in Daphnia magna. I. Selection and clonal diversity. Genetics, 92, 953-970.

YOUNG, J. P. W. 1979 b. Enzyme polymorphism and cyclic parthenogenesis in Daphnia magna. II. Heterosis following sexual reproduction. Genetics, 92, 971-982. 